\title{
UMA PRIMEIRA DESCRIÇÃO DA OBRA: RABDOLOGIAE, SEU NUMERATIONIS PER VIRGULA ... DE 1617
}

\section{A FIRST DESCRIPTION OF THE BOOK: RABDOLOGIAE, SEU NUMERATIONIS PER VIRGULA ... FROM 1617}

\author{
Eugeniano Brito Martins ${ }^{1}$ \\ Programa de Pós-graduação em Ensino de Ciências e Matemática \\ Instituto Federal de Educação, Ciência e Tecnologia do Ceará
}

Ana Carolina Costa Pereira ${ }^{2}$

Universidade Estadual do Ceará-UECE

\begin{abstract}
Resumo
Este trabalho é um recorte da dissertação que está sendo desenvolvida no Programa de Pós-Graduação em Ensino de Ciências e Matemática, do Instituto Federal de Educação, Ciências e Tecnologia do Ceará, cujo objetivo é utilizar um documento histórico para a identificação de potencialidades didáticas voltadas para o ensino, utilizando a história da matemática na formação de professores que lecionam esta disciplina na Educação Básica. Aqui é apresentado e descrito a obra Rabdologiae, Seu Numerationis Per Virgula ... (1617), escrita por John Napier (1550 - 1617), matemático escocês que inventou os diversos instrumentos descritos na obra. A pesquisa é qualitativa, focada no aspecto documental e descritivo da publicação. Nela, o autor apresenta a construção e utilização de três instrumentos cálculo que inventara, com a finalidade é agilizar o trabalho dos calculistas, reduzindo o tempo das contas e mantendo a precisão dos cálculos. Para isso, foi realizada uma pesquisa qualitativa descritiva da obra. Assim, diante desse estudo foi observado a estrutura da publicação, a língua em que foi escrita, o estilo de linguagem utilizado pelo autor e os conteúdos pertencentes a ela. Desta forma, percebe-se no documento estudado uma preocupação do autor, para com o leitor da época, na forma detalhada e ilustrada como descreve as soluções matemáticas no livro, tornando este livro em um manual para as pessoas que vão utilizar os instrumentos e fazer operações com os mesmos. Essa forma didática de escrever, possibilita a identificação de potencialidades didáticas associadas com o ensino de matemática, de modo, associativo com a História da Matemática.
\end{abstract}

Palavras-chave: Histórica da Matemática. Rabdologiae, seu Numerationis Per Virgula ... . Descrição da obra.

\begin{abstract}
This work is a part of the dissertation that is being developed in the Graduate Program in Science and Mathematics Teaching, of the Federal Institute of Education, Science and Technology of Ceará, whose aim is to use a historical document for the identification of didactic potentialities for teaching, using the history of mathematics in the training of teachers who teach this discipline in Primary and Secondary Education.
\end{abstract}

1 eugeniano.martins@ifce.edu.br

2 carolina.pereira@uece.br

Boletim Cearense de Educação e História da Matemática - Volume 05, Número 14, 154 - 166 (2018)

DOI: 10.30938/bocehm.v5i14.246 
Here it is presented and described the work Rabdologiae, Seu Numerationis Per Virgula ... (1617), written by John Napier (1550 - 1617), Scottish mathematics who invented the various instruments described in the work. The research is qualitative, focused on the documentary and descriptive aspect of the publication. In it, the author presents the construction and use of three calculation tools that he invented, with the purpose of speeding up the work of the arithmeticians, reducing the time of the accounts and keeping the precision of the calculations. For this, a descriptive qualitative research was carried out. Thus, from this study, it was observed the structure of the publication, the language in which it was written, the language style used by the author and the contents belonging to it. In this way, it is possible to notice in the studied document a concern from the author to the reader of the time, in the detailed and illustrated way he describes the mathematical solutions in the book, making this book a manual for people who will use the instruments and make operations with them. This didactic form of writing enables the identification of didactic potentialities associated with the teaching of mathematics, so associative with the History of Mathematics.

Keywords: Historical Mathematics. Rabdologiae, seu Numerationis Per Virgula ... . Description of the book.

\section{Introdução}

Uma forma de propiciar essa compreensão da construção dos conceitos matemáticos é a inserção de recursos didáticos que facilitem, em sala de aula, esse processo. A História da matemática é uma destas formas que possibilita compreender os diferentes conteúdos lecionados, sob a ótica dos fatores sociais e culturais das comunidades envolvidas. (MENDES, 2009). Dessa forma, estudos de Fauvel e Maanen (2000), Furinghetti e et al (2008), Miguel e Miorin (2004), mostram a que a interligação da história da matemática e o ensino pode ser realizado nos diversos conteúdos lecionados.

A utilização da história da matemática no ensino possui diversas motivações, algumas delas citadas por Miguel e Miorin (2004, p. 53):

(1) A matemática como uma criação humana; (2) as razões pelas quais as pessoas fazem Matemática; (3) as necessidades práticas, econômicas e físicas que servem de estímulo ao desenvolvimento das ideais matemáticas; (4) as conexões existentes entre matemática e filosofia, matemática e religião, matemática e lógica, etc.; (5) a curiosidade estritamente intelectual que pode levar a generalização e extensão de ideias e teorias; (6) as percepções que os matemáticos têm do próprio objeto da matemática, as quais mudam e se desenvolvem ao longo do tempo; (7) a natureza da uma estrutura, de uma axiomatização e de uma prova.

Os autores apresentam a articulação da história da matemática com o ensino, como uma forma de mudança no pensar dos professores, que mudando suas concepções, 
passariam a ver a matemática como uma construção humana, compreendendo que a razão do desenvolvimento de determinados conceitos, está relacionado com a sociedade e suas necessidades práticas, bem como, com suas relações comerciais, políticas e outras.

A formalização destes conhecimentos, dar-se baseado nas visões filosóficas presente na matemática, na religião, na lógica e, desta forma, influenciando o intelecto das pessoas envolvidas, que relacionam estes saberes a forma como o objeto é percebido no social e culturalmente. Assim, é possível ver a Matemática como uma construção humana, resultado das relações existentes na época da construção de seus conteúdos.

A História da Matemática, como recurso didático, possui diferentes formas de ser utilizada. Uma desta forma é com o uso de fontes históricas, estas são compreendidas como todo e qualquer material que represente a forma de pensar ou utilizar os conceitos matemáticos de uma época ou comunidade, conforme os pesquisadores, tais como Pinsky (2006), Silva (2013) e Saito (2015).

Assim, este trabalho, apresenta e descreve a obra matemática Rabdologiae, Seu Numerationis Per Virgula ... ${ }^{3}$, escrita no século XVII por John Napier e cuja a primeira publicação foi em 1617. Ela teve diversas publicações ao longo dos séculos XVII, XVIII e XIX, em diferentes países da Europa. Neste livro são apresentados três instrumentos de cálculo que serão melhor detalhados mais adiante.

\section{A fonte histórica e o ensino de matemática}

Fonte histórica é tudo que coloca o historiador em contato direto com seu problema, proporcionando material para ser examinado e analisado por uma sociedade humana ao longo do tempo. Estes necessitam serem pesquisados, decifrados, compreendidos, questionados, de modo a obter deles informações sobre o conhecimento do passado da humanidade.

Quando voltadas para o ensino de matemática, a utilização de fontes históricas em sala de aula está em desenvolvimento no Brasil. Porém, é necessário que a formação dos professores de matemática, contemple os conhecimentos necessários para possibilitar a utilização delas, quer sejam originais ou secundárias, como um recurso didático.

\footnotetext{
${ }^{3}$ O título completo da obra é: Rabdologiae, Seu Numerationis Per Virgulas Libri Duo: cum appendice de expeditíssimo Multiplicationes promptuario, quibus acessit e arithmeticea localis liber unus. 
Dentre os pesquisadores que fazem uso das fontes históricas para o ensino de matemática, destaca-se Jahnke (2002), Silva (2013), Pereira e Pereira (2015), entre outros. Silva (2013, p. 34), as define como sendo o "uso de textos que trazem à tona problemas que os sábios da antiguidade resolveram ou investigaram”.

Complementando, Saito (2015), estende essa definição para: livros, manuais, artefatos, instrumentos e construções que permitem a compreensão do pensamento que levaram ao desenvolvimento dos conceitos matemáticos utilizados atualmente.

A inserção de fontes históricas no ensino de matemática, na formação de professores possibilita que estes compreendam as potencialidades didáticas daquelas. Jahnke (2002), apresente alguns efeitos que são possíveis de se obter, quando da utilização daquelas como recursos didáticos. Tais como:

(i) substituição: a integração da história em matemática substitui o habitual por algo diferente: permite que a matemática seja vista como uma atividade intelectual, e não apenas como um corpus de conhecimento ou um conjunto de técnicas.

(ii) reorientação: a integração da história em matemática desafia as percepções de uma pessoa ao tornar o familiar desconhecido. Lidar com um texto histórico pode causar uma reorientação de nossos pontos de vista. A história da matemática tem a virtude de "surpreendente com o que vem de si mesmo (Veyne 1971)". Muitas vezes no ensino, o que acontece é que os conceitos aparecem como se já existissem. Isso é verdade para o conceito de um conjunto, por exemplo, mas tão verdadeiro para o conceito de um triângulo ou uma função. E os conceitos são manipulados sem pensar na sua construção. A história nos lembra que esses conceitos foram inventados e que isso não aconteceu sozinho.

(iii) compreensão cultural: a integração da história da matemática convidanos a colocar o desenvolvimento da matemática no contexto científico e tecnológico de um determinado momento e na história das ideias e sociedades e também considerar a história do ensino da matemática a partir de perspectivas que se situam fora dos limites disciplinares disciplinados estabelecidos. (JAHNKE, 2002, p. 292) ${ }^{4}$

\footnotetext{
4 (i) replacement: Integrating history in mathematics replaces the usual with something different: it allows mathematics to be seen as an intellectual activity, rather than as just a corpus of knowledge or a set of techniques.

(ii) reorientation: Integrating history in mathematics challenges one's perceptions through making the familiar unfamiliar. Getting to grips with a historical text can cause a reorientation of our views. History of mathematics has the virtue of 'astonishing with what comes of itself (Veyne 1971). All too often in teaching, what happens is that concepts appear as if already existing. This is true for the concept of a set, for example, but just as true for the concept of a triangle or a function. And concepts are manipulated with no thought for their construction. History reminds us that these concepts were invented and that this did not happen all by itself.

(iii) cultural understanding: Integrating history of mathematics invites us to place the development of mathematics in the scientific and technological context of a particular time and in the history of ideas and societies, and also to consider the history of teaching mathematics from perspectives that lie outside the established disciplinary subject boundaries.
} 
Desta forma, uma fonte histórica matemática permite entender o processo histórico de evolução desta Ciência e de seus conceitos, conforme as diferentes épocas históricas, considerando-se o contexto social e cultural de sua criação. Assim, a obra Rabdologiae, Seu Numerationis Per Virgula ..., enquadra como uma fonte histórica ao conter orientações para a construção de instrumentos de cálculos e sua utilização na resolução de problemas da era de sua escrita, possibilitando a articulação História e ensino.

\section{Metodologia}

Este trabalho apresenta uma pesquisa qualitativa, focando no aspecto documental e descritivo da obra Rabdologiae, Seu Numerationis Per Virgula ..., John Napier (1617). Este tipo de pesquisa trabalha com documentos que não passaram por um tratamento de análise ou crítica, caracterizando, assim, os arquivos primários, tais como, livros, documentos particulares, fotografias, vídeos, entre outros, que possibilitem fazer um reconhecimento mais detalhado das fontes escolhidas (GIL, 2002).

A escolha da obra deveu-se, inicialmente, ao fato de existir diversas pesquisas sobre a utilização do instrumento Barras Napier, voltadas ao ensino da multiplicação, porém desconectadas de uma abordagem histórica Lazarin (2004), Machado (2015) e outros. Em segundo lugar, o fato da obra ter sido publicada no início do século XVII, momento que se inicia a estruturação das ciências modernas e na preocupação de autor em encurtar os cálculos com a utilização de três instrumentos que inventou (NAPIER, 1617).

\section{Apresentação e descrição da obra}

A obra aqui apresentada, tem como título Rabdologiae, Seu Numerationis Per Virgulas Libri Duo: cum appendice de expeditíssimo Multiplicationes promptuario, quibus acessit e arithmeticea localis liber unus Rabdologiae ${ }^{5}$. A tradução do título seria: Rabdologia, dois livros de contar dinheiro com varetas: com um apêndice do prontuário de multiplicação muito rápida com a adição de um livro de Aritmética local, (Figura 1). Escrita pelo matemático escocês John Napier (1550-1617), como as obras do século XVII, apresenta em seu título uma descrição de os conteúdos que a compõem.

\footnotetext{
${ }^{5}$ Por praticidade, o título será abreviado para: Rabdologiae, Seu Numerationis Per Virgula ... Boletim Cearense de Educação e História da Matemática - Volume 05, Número 14, 154 - 166 (2018) DOI: $10.30938 /$ bocehm.v5i14.246
} 
A primeira publicação registada desta obra é em 1617, tendo sido organizada por Henry Briggs (1561 - 1630), após o falecimento do autor. Durante os séculos XVII, XVIII e XIX, diversas edições foram feitas, tanto na Inglaterra, como também, nos Países Baixos, França, Alemanha, Florença, Milão. Essas novas edições, tanto foram impressas em latim, como nas línguas locais de cada país, por exemplo, a uma edição de 1618, publicada em alemão.

Observando a capa da obra (Figura 1), ao final do título, o autor é apresentado como Authore e Inventore Ioanne Nepero, Barone Merchsitonii e Scoto (Autor e Inventor John Napier, Barão de Merchsitonii e Scoto), inventor pois na apresentação da obra ele afirma que: "no entanto, para o benefício daqueles que preferem operar com os números naturais, eu elaborei outros três métodos curtos de cálculo"6 (NAPIER, 1617, p. 3). Observa-se que os números que são trabalhados pelas invenções apresentadas na obra, já são chamados de números naturais. Ainda, observando a capa, o ano de impressão já está grafado em algarismos indo-arábicos, quando nesta época ainda era como utilizar-se algarismos romanos para tal finalidade.

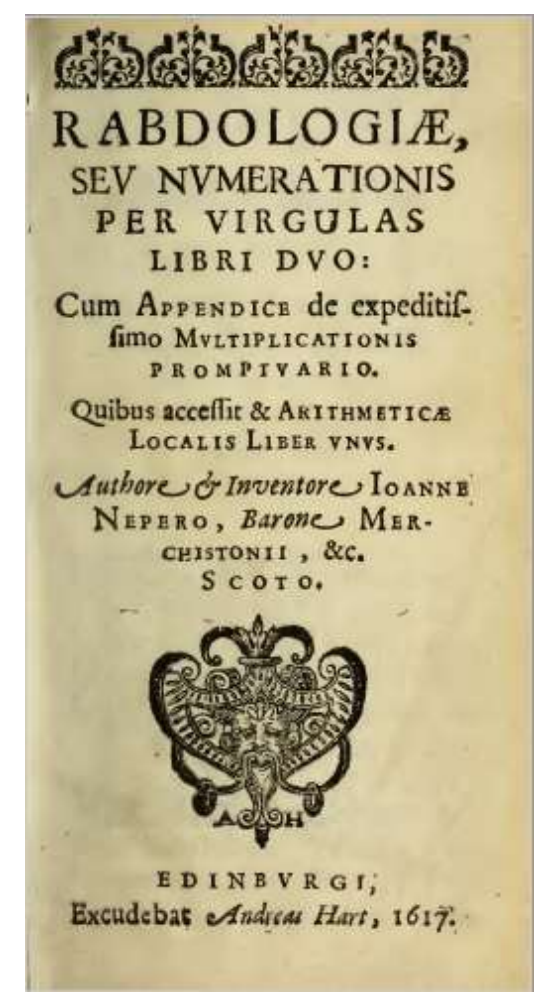

Figura 1: Capa do Rabdologiae, seu Numerationis per Virgulas ... (1617)

Fonte: Napier (1617)

\footnotetext{
${ }^{6}$ Intereà tamen in gratiam eorum qui per ipfos nurrieros naturaks oblatos operari maluerint, tria alia calculi compendia excogitavimus: quorum piimaim 
A edição do Rabdologiae, Seu Numerationis Per Virgula ..., apresentada neste trabalho, é a primeira impressão, realizada em 1617 (Figura 1), escrita em latim, John Napier apresenta a obra dizendo que "Para realizar cálculos (Nobre Senhor) o processo é longo e difícil, um tédio o qual atrapalha muitos dos estudos de Matemática. Sempre tentei, com tanta força e talento que possuo, para agilizar o processo"7 (NAPIER, 1617, p. 1).

A obra é constituída de quatro livros (Tabela 1). O Primeiro Livro descreve a construção e utilização das barras de números, conhecidas como barras Napier ou Ossos de Napier, pois era comum utilizar marfim em sua construção. No Segundo Livro, é apresentada a barra para cálculos geométricos. O terceiro livro, descreve a construção e utilização do tabuleiro mecânico, que é uma planificação das barras, com a finalidade de facilitar os cálculos. E o quarto contém os detalhes de construção e utilização do denominado do prontuário de multiplicação, conhecido, também, como ábaco de Napier.

Tabela 1. Descrição da obra Rabdologiae, Seu Numerationis Per Virgulas ....

\begin{tabular}{l|l|c|c}
\hline \multicolumn{1}{c|}{ LIVRO } & \multicolumn{1}{|c|}{ NOME DO LIVRO } & CAPÍTULOS & $\begin{array}{c}\text { TOTAL DE } \\
\text { PÁGINAS }\end{array}$ \\
\hline $\begin{array}{l}\text { Primeiro } \\
\text { Livro }\end{array}$ & $\begin{array}{l}\text { Em geral, a utilização das barras de } \\
\text { números de calcular }\end{array}$ & 9 & 42 \\
\hline $\begin{array}{l}\text { Segundo } \\
\text { Livro }\end{array}$ & $\begin{array}{l}\text { O Uso da Barra de Calcular Geométrico } \\
\text { ou Tabuleiro Mecânico. }\end{array}$ & 8 & 47 \\
\hline Apêndice & Um Prontuário de Multiplicação & 4 & 22 \\
\hline Último livro & Aritmética Local & 11 & 41 \\
\hline
\end{tabular}

Fonte: Napier (1617)

Ao apresentar o Rabdologiae, Seu Numerationis Per Virgula ..., Rice et al (2017), a identifica como a segunda publicação matemática de Napier e que "Como o Descriptio, foi dedicado a simplificar os cálculos com grandes números, mas os métodos propostos neste novo livro são radicalmente diferentes dos logaritmos" (p. 431).

\footnotetext{
${ }^{7}$ Diffitultatem \& prolixitatem calculi (Vir Ulustrissìme) cujus tedium purimos a studi Mathematutn deterrete folet, ego Semper prò viribus, \& ingerii modulo conatus fum è medio tollere.
} 
O primeiro livro, é apresentado a construção e utilização das barras de calcular, ou Barras de Napier ou Ossos de Napier (Figura 2). O livro possui 44 páginas, contendo 9 capítulos, assim denominados:

- Capítulo 1: Da fabricação e inscrição na barra, com 9 páginas;

- Capítulo 2: Aplicação das Barras aos números e o contrário, de 6 páginas;

- Capítulo 3: A Multiplicação, com 4 páginas;

- Capítulo 4: A divisão, com 5 páginas;

- Capítulo 5: Extração de raízes por varetas, com 2 páginas;

- Capítulo 6: A extração de raiz quadrada, 4 páginas;

- Capítulo 7: A extração de raiz cubica, com 6 páginas; $\square$ Capítulo 8: Um livro para extrair cubo, de 3 páginas;

- Capítulo 9: Regra de três direta e inversa, de 5 páginas.

Neste livro, observa-se a descrição e utilização das barras para a operações de multiplicação, divisão e a radiciação com raízes quadradas e cúbicas. Napier ao escrever a dedicatória, deixa claro que a publicação do livro sobre as barras é devido ao fato delas já serem bastante conhecidas e utilizadas tanto no seu país como no exterior. (NAPIER, 1617)

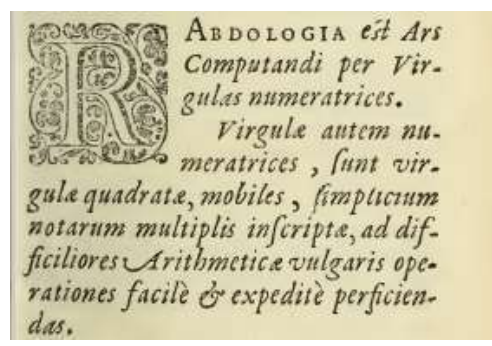

Figura 2: Parágrafos inicias do Capítulo $1^{8}$

Fonte: Napier (1617)

Observa-se ao longo dos capítulos a maneira didática de repassar as informações necessárias ao leitor para que este não apenas construa, como também, utilize as barras, como observado nos parágrafos iniciais do capítulo 1 (Figura 2). O formato delas é

\footnotetext{
${ }^{8}$ A Rabdologia é a arte de calcular por meio de barras com números nelas. Estas varas são quadradas e móveis e nelas estão inscritos os múltiplos de números simples. Eles são para se comportar fácil e rapidamente as operações mais difíceis da aritmética comum. Por isso, considero como fazer e usar estas hastes.
} 
apresentado na Figura 3, onde observamos a utilização de algarismos romanos para indicar cada lado da barra, destacando o número 4 é escrito como IIII.

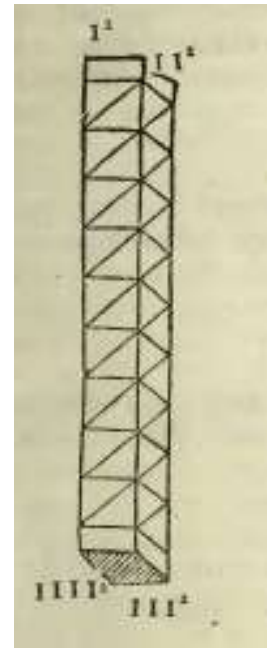

Figura 3: Desenho de uma barra de Napier.

Fonte: Napier (1617)

No segundo livro, é apresentado a utilização das barras para realização de cálculos de área, diâmetros e volume, apresentando uma forma diferente da apresentada no livro anterior, para a aplicação das barras, como observável na Tabela 2, a seguir.

Tabela 2. Descrição do segundo livro do Rabdologiae, Seu Numerationis Per Virgulas

\begin{tabular}{c|l|c}
\hline CAPÍTULO & \multicolumn{1}{|c}{ NOME DO CAPÍTULO } & TOTAL DE PÁGINAS \\
\hline I & Tabelas de descrição de frequências. & 2 \\
\hline II & $\begin{array}{l}\text { A invenção de tijolos de polígonos } \\
\text { quadratrice, os quatro problemas. }\end{array}$ & 8 \\
\hline III & $\begin{array}{l}\text { A descoberta do quadratrice, a partir dos } \\
\text { diâmetros do polígono, os quatro problemas. }\end{array}$ & 7 \\
\hline IV & $\begin{array}{l}\text { Da determinação dos diâmetros de fora dos } \\
\text { lados de um polígono, os quatro problemas. }\end{array}$ & 5 \\
\hline V & $\begin{array}{l}\text { Achando o volume de corpos regulares, os } \\
\text { quatro problemas. }\end{array}$ & 5 \\
\hline VI & $\begin{array}{l}\text { A invenção do diâmetro e do volume da esfera, } \\
\text { os quatro problemas. }\end{array}$ & 5 \\
\hline VII & $\begin{array}{l}\text { A partir dos lados das figuras regulares, os } \\
\text { quatro problemas. }\end{array}$ & 5 \\
\hline VIII & $\begin{array}{l}\text { Como descobrir pedras da magnitude dos } \\
\text { pesos, os quatro problemas. }\end{array}$ & \multicolumn{2}{|c}{5} \\
\hline
\end{tabular}

Fonte: Napier (1617) 
Neste livro, é apresentado uma versão planificada da barra de modo que os resultados são obtidos de forma mais rápida do que nas barras.

O terceiro livro da obra, é denominado por John Napier com o nome de Apêndice, para Rice e et al (2017), demonstra que o autor antes de concluir o livro, teve uma ideia brilhante e a inseriu nesta posição. Ele é dividido em 4 capítulos, que são descritos a seguir:

- $\quad$ Prefácio (1 página)

- $\quad$ Capítulo 1: Como fabricar os Prontuários de placas. (6 páginas)

- Capítulo 2: Placas para preservar as estruturas do Prontuário. (5 páginas)

- Capítulo 3: O prontuário para facilitar a multiplicação. (7 páginas)

- $\quad$ Capítulo 4: A divisão pelo prontuário e as tabelas. (5 páginas)

Neste apêndice, estão colocadas três páginas que contém um esquema detalhado sobre como construir e utilizar essas placas para as operações de multiplicação e divisão. Diferentemente do que se tem no primeiro livro, aqui não são barras que se utiliza, mas sim chapas planas onde números são inseridos e fitas são utilizadas para obter os produtos e os quocientes.

Finalizado a obra, tem-se o quarto livro, denominado por Napier como Aritmética Local, composto de onze capítulos, distribuídos em 45 páginas, conforme detalhado na Tabela 4.

Tabela 3. Descrição do Quarto Livro do Rabdologiae, Seu Numerationis Per Virgulas

\begin{tabular}{c|l|c}
\hline CAPÍTULO & \multicolumn{1}{|c}{ NOME DO CAPÍTULO } & TOTAL DE PÁGINAS \\
\hline I & Descrição das praticas lineares locais. & 2 \\
\hline II & Das transações com números do povo local. & 3 \\
\hline III & Da redução numérica do povo local. & 1 \\
\hline IV & Uma versão resumida. & 3 \\
\hline V & $\begin{array}{l}\text { Da adição e subtração e da transferência dos } \\
\text { ganhos da redução. }\end{array}$ & 2 \\
\hline VI & $\begin{array}{l}\text { A descrição do ábaco, ou canais, em vez de } \\
\text { deixar solto. }\end{array}$ & \\
\hline
\end{tabular}




\begin{tabular}{c|l|c}
\hline VII & O movimento dos números na tabela. & 2 \\
\hline VIII & Axioma e conjectura do movimento na tabela. & 4 \\
\hline IX & Da multiplicação. & 7 \\
\hline X & Da divisão. & 7 \\
\hline XI & Da extração da raiz quadrada. & 7 \\
\hline
\end{tabular}

Fonte: Napier (1617)

O último livro apresenta um ábaco, denominado por Napier, como tabuleiro de xadrez. Com este instrumento é possível fazer somas, subtrações, multiplicações divisões e extração de raízes quadradas, utilizando o princípio de que todo número pode ser representado como uma potência de base 2 . $\mathrm{O}$ autor apresenta as diversas formas de realizar essas operações utilizando problemas do cotidiano.

$\mathrm{Na}$ resolução de todos os exemplos e problemas, que ao longo da obra o autor utiliza os instrumentos por ele criado, encontram-se presentes no ensino de matemática. Desta forma, existem potencialidades didáticas a serem identificadas que contribuam para o ensino, ao explorar a fonte histórica e permitindo, assim, inserir a história da matemática.

\section{Considerações finais}

Utilizar fontes históricas como recurso didático, em aulas de matemática, possibilita explorar os diversos conteúdos lecionados da forma que se encontra inserida dentro da obra. Desta forma, é possível articular os conhecimentos matemáticos com o contexto social, cultural, político, religioso e outras, que levaram ao estudo destes conceitos ao longo do tempo da obra até o momento presente.

Com a realização deste estudo sobre o Rabdologiae, Seu Numerationis Per Virgula ..., percebe-se que foi uma obra importante em sua época, ao divulgar instrumentos de cálculos que agilizavam a sua realização, divulgando o processo de construção e utilização destes artefatos, ao mesmo tempo que popularizou os processos de resolução de problemas presente no cotidiano e que atravessaram os séculos até nossa época.

O fato dos conteúdos matemáticos e dos problemas apresentados na obra estudada fazerem-se presente no cotidiano atual, torna-a potencialmente didática para 
ser utilizada em sala de aula, para a mobilização dos conhecimentos matemáticos presente na construção dos instrumentos e na resolução dos problemas.

Desta forma, está sendo dada continuidade à pesquisa, com a tradução da fonte de modo a manter-se fiel às ideias do autor, visando identificar as potencialidades didáticas presentes na obra.

\section{Referências}

FAUVEL, J.; MAANEN, J. V. History in mathematics education: the ICMI study. Dordrecht: Kluwer Academic. 2000.

FURINGHETTI, F. et al. The role of the history of mathematics in mathematics education. In: INTERNATIONAL CONGRESS ON MATHEMATICAL EDUCATION, 11., 2008, Monterrey. Proceedings. Monterrey, 2008. v. 1, p. 1 - 4. Disponível em: <http://www.mathunion.org/fileadmin/ICMI/files/Digital_Library/ICMEs/TSG_23_Rep ort_BB_FF.pdf >. Acesso em: 11 jul. 2017.

GIL, Antonio Carlos. Como elaborar projetos de pesquisa. 4. ed. São Paulo: Editora Atlas, 2002. $175 \mathrm{p}$.

JAHNKE, Hans Niels. The use of original sources in the mathematics classroom. In: FAUVEL, John; VAN MAANEN, Jan. History in Mathematics Education. New York, Boston, Dordrecht, London, Moscow: Kluwer Academic Publishers, 2002. Cap. 9. p. 291-328.

LAZARIN, Zélia Bavaresco. Ossos de Napier e Réguas de Genaille-Lucas. 2004. 89 f. Trabalho de Conclusão de Curso (Licenciatura em Matemática). Universidade Federal de Santa Catarina. 2004.

MACHADO, Benedito Fialho. Confecção de artefatos de cálculos antigos em sala de aula: o ábaco de Napier. In: Seminário Nacional de História da Matemática, XI, 2015, Natal. Comunicação ..., Natal: Universidade Federal do Rio Grande do Norte, 2015 .

MENDES, Iran Abreu. Investigação Histórica no Ensino da Matemática. Rio de Janeiro: Ciência Moderna, 2009. 256 p.

MIGUEL, A; MIORIN, M. A. História na Educação Matemática: propostas e desafios. Belo Horizonte: Autêntica, 2004. 200 p.

PINSKY, C. B. (org). Fontes Históricas. São Paulo: Contexto, 2006.

NAPIER, John. Rabdologiae, Seu Numerationis Per Virgulas Libri Duo: cum appendice de expeditíssimo Multiplicationes promptuario, quibus acessit e arithmeticea localis liber unus. Edinburg: Andreas Hart, 1617. Obtida em http://archive.org/details/rabdologiae00napi; em janeiro de 2017 
PEREIRA, Ana Carolina Costa; PEREIRA, Daniele Esteves. Ensaio sobre o uso de fontes históricas no ensino de Matemática. REMATEC. Revista de Matemática, Ensino e Cultura (UFRN), v. 10, p. 65-78, 2015.

RICE, Brian; GONZÁLEZ-VELASCO, Enrique; CORRIGAN, Alexander. The Life and Works of John Napier. Suiça:Springer ,2017. 1009 p

SAITO, Fumikazu. História da matemática e suas (re)construções contextuais. São Paulo: Editora Livraria da Física, 2015. 259 p.

SILVA, Ana Paula Pereira do Nascimento. A leitura de fontes antigas e a formação de um corpo interdisciplinar de conhecimentos: Um exemplo a partir do Almagesto de Ptolomeu. 2013. 100 f. Dissertação (Mestrado em Ensino de Ciências Naturais e Matemática) - Universidade Federal do Rio Grande do Norte, Natal, 2013. 